\title{
Path Integral Solution of PT-/non-PT-Symmetric and non-Hermitian Hulthen Potential
}

\author{
N. Kandirmaz, R. Sever
}

\begin{abstract}
The wave functions and the energy spectrum of PT-/non-PT-Symmetric and non-Hermitian Hulthen potential are of an exponential type and are obtained via the path integral. The path integral is constructed using parametric time and point transformation.
\end{abstract}

Keywords: PT-symmetry, coherent states, path integral, Hulthen Potential.

\section{Introduction}

A suggestion by Bender and Boetcher on PTsymmetric quantum mechanics has put forward a different point of view from standard quantum mechanics. For a quantum mechanical system have to a real energy spectrum, the Hamiltonian must be Hermitian. Bender and his co-workers showed that even if a Hamiltonian is not Hermitian, it has a real energy spectrum [1]. PT-symmetric and non Hermitian potentials have been studied to prove they have a real energy spectrum, using numerical and analytical techniques. The energy spectrum corresponding to the wave functions is also calculated [2-9].

In this work, we have used Feynman's path integral method to get the energy spectrum and the wave functions of the PT-/Non-PT-Symmetric and nonHermitian exponential potential. The Feynman Path Integral is a given kernel which has transition amplitudes between the initial and final positions of the energy dependent Green function. A Feynman Path Integral formalism for deriving the kernel of various potentials was developed in [10-16]. Duru derived the wave functions and the energy spectrum of the Wood-Saxon potential for s-waves via the radial path integral. Inomata obtained the energy spectrum and the normalized s-state eigenfunctions for the Hulthen Potential using the Green function [11]. The kernel of the Hulthen potential can be exactly solved given the path integral for the particle motion on the $\mathrm{SU}(2)$ manifold $S^{3}$ [10-12]. In Sec. II and III, we derive the energy dependent Green's function of the PT/Non-PT-Symmetric and non-Hermitian q-deformed Hulthen Potential. We obtained the energy eigenvalues and the corresponding wave functions.

\section{PT-Symmetric and Non-Hermitian Hulthen Potential}

The kernel of a point particle moving in the $V(x)$ potential in one dimension is represented by the following path integral

$$
\begin{aligned}
K\left(x_{b}, t_{b} ; x_{a}, t_{a}\right)= & \int \frac{D x D p}{2 \pi} . \\
& \exp \left\{i \int \mathrm{d} t\left[p \dot{x}-\frac{p^{2}}{2 m}-V(x)\right]\right\}
\end{aligned}
$$

where $\hbar=1$. The kernel expresses the probability amplitude of a particle moving to position $x_{b}$ at time $t_{b}$ from position $x_{a}$ at time $t_{a}$. The time interval can be divided into $n$ equal parts

$$
t_{j}-t_{j-1}=t_{b}-t_{a}=T \quad j=1,2,3, \ldots, N
$$

and taking initial position is $x_{a}$ and final position $x_{b}$, the kernel [11] can be performed as

$$
\begin{aligned}
& K\left(x_{b}, T ; x_{a}, 0\right)=\int_{-\infty}^{\infty} \prod_{i=1}^{n} \mathrm{~d} x_{i} \prod_{i=1}^{n+1} \frac{\mathrm{d} p_{i}}{2 \pi} \\
& \exp \left\{i \sum_{i=1}^{n+1}\left[p_{i}\left(x_{i}-x_{i-1}\right)-\frac{p_{i}^{2}}{2 m}-V\left(x_{i}\right)\right]\right\}
\end{aligned}
$$
is

The PT- symmetric and non-Hermitian potential

$$
V(x)=-\frac{V_{o} e^{-i x / a}}{1-q e^{-i x / a}}
$$

which is determined by taking $\frac{1}{a} \longrightarrow \frac{i}{a}$ in the $q$-deformed Hulthen potential [5].

We will start by applying point transformation to get a solvable path integral form for the Hulthen potential

$$
\frac{1}{1-q e^{-i x / a}}=\sin ^{2} \theta \quad p=\frac{i}{2 a} \sin \theta \cos \theta p_{\theta}
$$


Because of this transformation, there is a contribution to the Jacobi performed kernel

$$
\begin{aligned}
& K\left(x_{b}, T ; x_{a}, 0\right)=\frac{q}{2 a} \sin \theta_{b} \cos \theta_{b} \int D \theta D p_{\theta} \times \\
& \exp \left[i \int \mathrm{d} t\left(p_{\theta} \dot{\theta}+\frac{\sin ^{2} \theta \cos ^{2} \theta}{4 \alpha^{2}} \frac{p_{\theta}^{2}}{2 \mu}-V_{0} \cos ^{2} \theta\right)\right] .
\end{aligned}
$$

Here, the kinetic energy term becomes positive. We define a new time parameter $s[12]$ to eliminate the $\frac{\sin ^{2} \theta \cos ^{2} \theta}{4 \alpha^{2}}$ part in the kinetic energy term

$\frac{\mathrm{d} t}{\mathrm{~d} s}=-\frac{4 a^{2}}{\sin ^{2} \theta \cos ^{2} \theta}$ or $t=-4 a^{2} \int \frac{\mathrm{d} s^{\prime}}{\sin ^{2} \theta \cos ^{2} \theta}$.

If we use the Fourier transform of the $\delta$ - function, we can write

$$
\begin{aligned}
1= & \int \mathrm{d} S \int \frac{\mathrm{d} E}{2 \pi} \frac{4 a^{2}}{\sin ^{2} \theta_{b} \cos ^{2} \theta_{b}} \cdot \\
& \exp \left[-i\left(E T-\int \mathrm{d} s \frac{4 a^{2} E}{\sin ^{2} \theta \cos ^{2} \theta}\right)\right]
\end{aligned}
$$

where $S=s_{b}-s_{a}$.

The factor in front of the path integral reached from the Jacobian can be a symmetrization according to points $a$ and $b$, as follows

$$
\begin{aligned}
\frac{1}{\sin \theta_{b} \cos \theta_{b}}= & \frac{2}{\sqrt{\sin 2 \theta_{a} \sin 2 \theta_{b}}} \cdot \\
& \exp \left(i \int_{0}^{S} \mathrm{~d} s(-i) \frac{\cos 2 \theta}{\sin 2 \theta} \dot{\theta}\right)
\end{aligned}
$$

Thus Eq. (6) happens

$$
\begin{aligned}
K\left(x_{b}, x_{a}, T\right)= & \int_{0}^{\infty} \mathrm{d} S e^{i S / 2 \mu} \int_{-\infty}^{\infty} \frac{\mathrm{d} E}{2 \pi} e^{i E T} . \\
& \frac{4 i a q}{\sqrt{\sin 2 \theta_{a} \cos 2 \theta_{b}}} K\left(\theta_{b}, \theta_{a} ; S\right)
\end{aligned}
$$

where

$$
\begin{aligned}
& K\left(\theta_{b}, \theta_{a} ; S\right)=\int D \theta D p_{\theta} . \\
& \exp \left\{i \int _ { 0 } ^ { S } \mathrm { d } s \left[p_{\theta} \dot{\theta}-\frac{p_{\theta}^{2}}{2 \mu}-\right.\right. \\
& \left.\left.\frac{1}{2 \mu}\left(\frac{K(K-1)}{\sin ^{2} \theta}+\frac{\lambda(\lambda-1)}{\cos ^{2} \theta}\right)-\frac{i p_{\theta} \cos 2 \theta}{2 \mu \sin 2 \theta}\right]\right\}
\end{aligned}
$$

and $K$ and $\lambda$ are

$$
\begin{aligned}
K & =\frac{1}{2}\left[1+\sqrt{32 \mu a^{2}\left(V_{0}+E\right)}\right] \\
\lambda & =\frac{1}{2}\left[1+\sqrt{32 \mu a^{2} E}\right]
\end{aligned}
$$

if the factor contribution to the Jacobian is symmetrized as [11] the contributions to the kernel become

$$
\dot{\theta_{j}} \longrightarrow \dot{\theta}_{j} \pm \frac{i \cos \theta_{j}}{2 \mu \sin \theta_{j}}
$$

So the problem is transformed into the path integral for Pöschl-Teller potential, for which an exact solution is known [11]. $K\left(\theta_{b}, \theta_{a} ; S\right)$ can be obtained as

$$
\begin{aligned}
& K\left(\theta_{b}, \theta_{a} ; S\right)=\int D \theta D p_{\theta} . \\
& \exp \left\{i \int _ { 0 } ^ { S } \mathrm { d } s \left[p_{\theta} \dot{\theta}-\frac{p_{\theta}^{2}}{2 \mu}-\right.\right. \\
& \left.\left.\frac{1}{2 \mu}\left(\frac{K(K-1)}{\sin ^{2} \theta}+\frac{\lambda(\lambda-1)}{\cos ^{2} \theta}\right)\right]\right\}
\end{aligned}
$$

The kernel can be obtained in the form

$$
\begin{aligned}
& K\left(\theta_{b}, \theta_{a} ; S\right)= \\
& \sum_{n=0}^{\infty} \exp \left[-i(S / 2 \mu)(K+\lambda+2 n)^{2}\right] \psi_{n}\left(\theta_{a}\right) \psi_{n}^{*}\left(\theta_{b}\right)
\end{aligned}
$$

where

$$
\begin{aligned}
\psi_{n}(\theta)= & \sqrt{2(K+\lambda+2 n)} . \\
& \sqrt{\frac{\Gamma(n+1) \Gamma(K+\lambda+n)}{\Gamma\left(\lambda+n+\frac{1}{2}\right) \Gamma\left(K+n+\frac{1}{2}\right)}} \times \\
& (\cos \theta)^{\lambda}(\sin \theta)^{K} P_{n}^{(K-1 / 2, \lambda-1 / 2)} . \\
& \left(1-2 \sin ^{2} \theta\right)
\end{aligned}
$$

With integrating over $\mathrm{d} S$, the Green's function for the Hulthen potential can be obtained as

$$
\begin{aligned}
& G\left(x_{b}, x_{a} ; E\right)=\frac{8 \mu a q}{\sqrt{\sin 2 \theta_{a} \cos 2 \theta_{b}}} \\
& \sum_{n=0}^{\infty} \int_{-\infty}^{\infty} \frac{\mathrm{d} E}{2 \pi} \frac{e^{i E T}}{(K+\lambda+2 n)^{2}-1} \psi_{n}\left(\theta_{a}\right) \psi_{n}^{*}\left(\theta_{b}\right)
\end{aligned}
$$

Therefore, the kernel of the physical system is rewritten as

$$
\begin{aligned}
K\left(x_{b}, x_{a} ; E\right)= & \sum_{n=0}^{\infty} e^{-i E_{n} T} \varphi_{n}\left(x_{a}\right) \varphi_{n}^{*}\left(x_{b}\right)= \\
& \sum_{n=0}^{\infty} \exp \left\{\left[-\frac{1}{8 \mu a q(n+1)^{2}} .\right.\right. \\
& {\left.\left.\left[(n+1)^{2}+2 \mu a^{2} V_{0}\right]^{2}\right] T\right\} . } \\
& \phi_{n}\left(u_{b}\right) \phi_{n}^{*}\left(u_{a}\right) .
\end{aligned}
$$

Integrating over $d E$, we can get the energy eigenvalues

$$
E_{n}=\frac{1}{8 \mu a^{2}(n+1)^{2}}\left[2 \mu a^{2} \frac{V_{0}}{q}-(n+1)^{2}\right]^{2}
$$


and the normalized wave functions in terms of Jacobi polynomials are

$$
\begin{aligned}
& \phi(x)=\frac{1}{2 \sqrt{2} \sqrt{n+1}} \sqrt{4(n+1)^{2}-\left(\lambda_{n}-K_{n}\right)^{2}} . \\
& \sqrt{\frac{\Gamma(n+1) \Gamma\left(K_{n}+\lambda_{n}+n\right)}{\Gamma\left(\lambda_{n}+n+\frac{1}{2}\right) \Gamma\left(K_{n}+n+\frac{1}{2}\right)}} \times \\
& \frac{\exp \left[\left(K_{n}-1 / 2\right) x / 2 a\right]}{\left(1+e^{-x / a}\right)} P_{n}^{\left(K_{n}+\lambda_{n}-1 / 2\right)} \\
& \left(-\frac{1+e^{-i x / a}}{1-e^{-i x / a}}\right)
\end{aligned}
$$

where we got

$$
\begin{aligned}
K_{n} & =\frac{1}{8 \mu a^{2}(n+1)^{2}}\left[(n+1)^{2}-2 \mu a^{2} \frac{V_{0}}{q}\right] \\
\lambda_{n} & =\frac{1}{2}+\frac{1}{n+1}\left[(n+1)^{2}+2 \mu a^{2} \frac{V_{0}}{q}\right]
\end{aligned}
$$

Here we see that the PT Symmetric and NonHermitian Hulthen potential has real energy spectra.

\section{Non- PT-symmetric and non-Hermitian Hulthen Potential}

The non PT-symmetric and non Hermitian Hulthen potential is determined by taking $\frac{1}{a} \rightarrow \frac{i}{a}, V_{0} \rightarrow$ $A+i B$ and $q \rightarrow i q$ as

$$
V(x)=-\frac{i V_{o} e^{-i x / a}}{1-i q e^{-i x / a}}
$$

We will follow the same steps for getting the wave function and the energy spectrum. A suitable coordinate transformation kernel is obtained as

$$
\begin{aligned}
& K\left(x_{b}, T ; x_{a}, 0\right)=\frac{q}{2 a} \sin \theta_{b} \cos \theta_{b} \int D \theta D p_{\theta} \cdot \\
& \exp \left[i \int \mathrm{d} t\left(p_{\theta} \dot{\theta}-\frac{\sin ^{2} \theta \cos ^{2} \theta}{4 \alpha^{2}} \frac{p_{\theta}^{2}}{2 \mu}-V_{0} \cos ^{2} \theta\right)\right] .
\end{aligned}
$$

If we follow the steps in sec. (2), we will obtain energy eigenvalues

$$
\begin{aligned}
E_{n}= & \frac{1}{8 \mu a^{2}(n+1)^{2}} . \\
& {\left[(n+1)^{2}+2 \mu a^{2} \frac{(i A-B)}{q}\right]^{2} }
\end{aligned}
$$

and the normalized wave functions in terms of Jacobi polynomials are

$$
\phi(x)=\frac{1}{2 \sqrt{2} \sqrt{n+1}} \sqrt{4(n+1)^{2}-\left(\lambda_{n}-K_{n}\right)^{2}} .
$$

$$
\begin{aligned}
& \sqrt{\frac{\Gamma(n+1) \Gamma\left(K_{n}+\lambda_{n}+n\right)}{\Gamma\left(\lambda_{n}+n+\frac{1}{2}\right) \Gamma\left(K_{n}+n+\frac{1}{2}\right)}} \times \\
& \frac{\exp \left[\left(K_{n}-1 / 2\right) x / 2 a\right]}{\left(1+e^{x / a}\right)^{\left(K_{n}+\lambda_{n}-1 / 2\right)}} . \\
& P_{n}^{\left(K_{n}-1 / 2, \lambda-1 / 2\right)}\left(\frac{1-e^{-x / a}}{1+e^{-x / a}}\right)
\end{aligned}
$$

$K_{n}$ and $\lambda_{n}$ are the same in Eq. (21). It is clear that the energy spectra are real only if $\operatorname{Re}\left(V_{0}\right)=0$.

\section{Conclusion}

We have calculated the energy eigenvalues and the corresponding wave functions for the PT-/non-PT Symmetric and non-Hermitian Deformed Hulthen Potential. We obtained that PT-/non-PT Symmetric and non-Hermitian forms of potentials have real energy spectra by restricting the potential parameters.

\section{References}

[1] Bender, C. M.: Reports on Progress In Physics 70(6), 947-1018 (2007); Bender, C. M., Darg, D. W.: Journal of Mathematical Physics 48(4), 042703 (2007); Bender, C. M., Boetcher, S.: Pys. Lett. 80, 5243 (1998); Bender, C. M., Boetcher, S., Meisenger, P. N.: J. Math. Phys. 40, 2201 (1999); Bender, C. M., Dunne, G. V., Meisenger, P. N.: Phys. Lett. A, 252, 272 (1999).

[2] Yesiltas, O., Simsek, M., Sever, R., Tezcan, C.: Phys. Scripta, T67, 472 (2003).

[3] Berkdemir, C., Berkdemir, A., Sever, R.: Phys. Rev. C72, 027001 (2005).

[4] Faridfathi, G., Sever, R., Aktas, M.: J. Math. Chem. 38, 533 (2005).

[5] Egrifes, H., Sever, R.: Int. J. Theo. Phys. 46, 935 (2007).

[6] Ikhdair, S. M., Sever, R.: J. of Math. Chem. 42, 461 (2007).

[7] Ikhdair, S. M., Sever, R.: J. Mod. Phys. E 17, 1107 (2008).

[8] Kandirmaz, N., Sever, R.: Chinese J. Phys. 47, 47 (2009).

[9] Kandirmaz, N., Sever, R.: Physica Scripta, 87, $3,(2010)$.

[10] Feynmann, R., Hibbs, A.: Quantum Mechanics and Path Integrals. McGraw Hill, New York, (1965). 
[11] Duru, I. H.: Phys. Rev. D, 28, 2689 (1983); Duru, I. H., Kleinert, H.: Phys. Lett. B84, 185 (1979); Fortschr. Phys. 30, 401 (1982); Duru, I. H.: Phys.Rev. D, 30, 2121 (1984); Duru, I. H.: Phys. Lett A, 119, 163 (1986).

[12] Peak, D., Inomata, A.: J. Math. Phys. 10, 1422 (1969).

[13] Erkoc, S., Sever, R.: Phys. Rev D, 30, 2117 (1984).

[14] Erkoc, S., Sever, R.: Phys. Rev A, 37, 2687 (1988).

[15] Pak, N. K., Sokmen, I.: Phys. Lett. 100A, 327 (1984).
[16] Gradshteyn, I. S., Ryzhic, I. M.: Table of Integrals, Series, and Products, 2nd ed., Academic Press, New York, (1981).

Nalan Kandirmaz

E-mail: nkandirmaz@mersin.edu.tr

Mersin University

Department of Physics

Mersin, Turkey

Ramazan Sever

Middle East Technical University

Department of Physics

Ankara, Turkey 